\title{
FORMATION OF BREMSSTRAHLUNG FLOW WITH SMALL DIVERGENCE AT LINAC OUTPUT FOR PLANET SURFACE SOUNDING WITH INTERPLANETARY SPACE STATIONS
}

\author{
B. Bogdanovitch $^{1}$, V. Kudinov, S. Minaev, A. Nesterovitch, \\ Yu.Pomazan, MEPhI, Moscow, Russia.
}

\begin{abstract}
The task of atmosphere structure or planet surface definition from interplanetary space stations can be decided both with use of hydrogen neutral atoms beams [1], and flows of brake radiation. One case depth of an analysed layer is much higher in view of greater penetrating ability of quantums in comparison with neutral particles. The brake radiation is used by search of minerals with the definition from interplanetary space stations accelerators help. Till now space geophysics development restrained by radiation large angular divergence, caused target significant thickness at the accelerator. In works [2-3] the research results of various ways of electron flows radiation formation, driven on a cyclic or spiral orbit were stated. The opportunity of a radiation intensity increase due to electron multifold passage of the thin target under condition at the electron flow adiabatic cooling was experimentally confirmed.

The mane difference of these modes was the maximum energy and minimum radiation flow divergence restriction caused by realising design specificity. The research results of system intended for electron beams with energy up to several hundreds $\mathrm{MeV}$ and angular divergence (110) $\mathrm{mrad}$ are described in the paper given.
\end{abstract}

\section{THE BREMSSTRAHLUNG FLOW FORMATION SCHEME}

The device scheme realising an proposed method in the specified parameters region, is shown in a Fig. 1.

To avoid attenuation of radiation in a target and to increase an electron beam use efficiency, it is proposed to set a sequence of thin targets (foils) in periodic magnetic system (PMS) with a vertical rejecting magnetic field with changing direction (the "wiggler" type system). If to place thin targets in each second interval between magnets, i.e. in points with maximal on value and identical on sign by an beam trajectory angular deviation, the radiation from each previous targets within the limits of some angular divergence will pass all next foils, and the beam incidence direction on each target can be made identical. Hence, in such system the effect of generated radiation attenuation in a thick target will be excluded with remaining of separate radiation vectors parallelism.

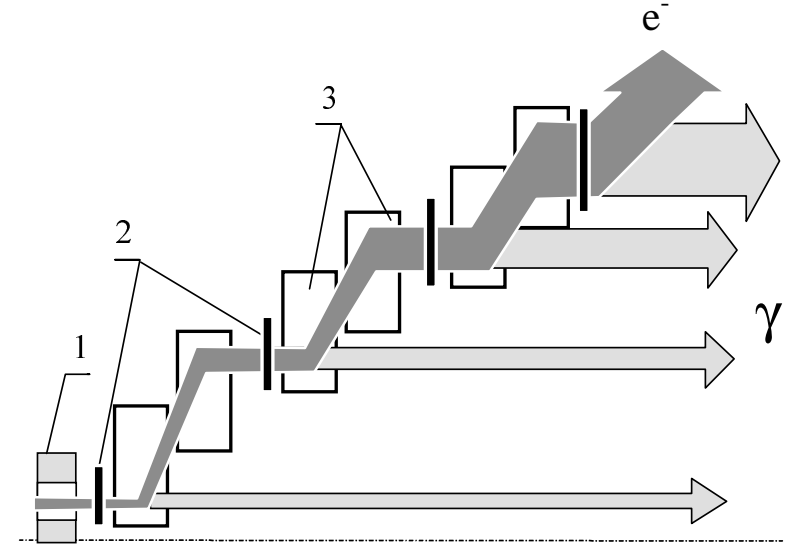

Figure 1: The bremsstrahlung flow formation scheme (1 - electron accelerator; 2 - foils; 3 magnets)

The single target thickness defines as well radiation angular divergence. With passage of an electron beam through substance the electron angular disorder stochastically grows, so beam cross temperature and, accordingly, it's emittance are increasing. It is obvious, that thus bremsstrahlung photons, produced in the target depth, also have a wider angular spectrum. Thus, if a subject of interest is the energy irradiated within a small cone angle, it is possible to tell, that the beam use efficiency on a thick target decreases not only due to attenuation in deep layers, but also because of the electron angular disorder increase.

The PMS with thin targets proposed can partially compensate this effect. The beam angular disorder is increased by jump after each target passing. If in PMS it was possible to provide effective simultaneous beam focusing in both cross directions (vertical and horizontal), on each site between the previous and subsequent targets it would be possible to reduce a little a beam angular divergence in comparison to equivalent uniform thick target. Certainly, thus it is impossible to prevent or to reduce beam cross emittance growth due to stochastic processes of dispersion in substance. It is possible to hope only for some reduction of angular divergence by the cross sizes increase. So with the successfully chosen PMS with thin targets parameters the electron beam can have smaller angular divergence, but more large cross sizes in comparison to a thick target.

${ }^{1}$ E-mail: bogdan@uni.mephi.ru 


\section{BREMSSTRAHLUNG PHOTONS OUTPUT FROM THE TARGET}

The expression for bremsstrahlung radiation angular distribution from thick targets can be received by taking into account repeated dissipate electron distribution on the target depth. Lawson, Lanzl and Hanson used for it distribution Moliere in the form: $\exp \left(-E_{0}{ }^{2} \theta^{2} / c t\right)$. A constant $c$ chose so that distribution width was same, that exact expression. The repeated dissipate electron distribution convolution and bremsstrahlung radiation angular distribution for an indefinitely thin target, integrated by complete thickness, gives the following expression for bremsstrahlung radiation angular distribution from a target thickness $t$ :

$$
\begin{gathered}
F\left(\theta, E_{0}\right)=F_{1}\left(\theta, E_{0}\right)+F_{2}\left(\theta, E_{0}\right) \\
F_{1}\left(\theta, E_{0}\right)=\frac{0.85 \theta_{1}^{2}\left(-E i\left(-\frac{E_{0} \theta^{2}}{c t+\theta_{1}^{2}}\right)+E i\left(-\frac{E_{0}^{2} \theta^{2}}{\theta_{1}^{2}}\right)\right)}{0.85 \theta_{1}^{2} \ln \left(1+\frac{c t}{\theta_{1}^{2}}\right)+0.15 \theta_{2}^{2} \ln \left(1+\frac{c t}{\theta_{2}^{2}}\right)} \\
F_{2}\left(\theta, E_{0}\right)=\frac{0.85 \theta_{2}^{2}\left(-E i\left(-\frac{E_{0} \theta^{2}}{c t+\theta_{2}^{2}}\right)+E i\left(-\frac{E_{0}^{2} \theta^{2}}{\theta_{2}^{2}}\right)\right)}{0.85 \theta_{1}^{2} \ln \left(1+\frac{c t}{\theta_{1}^{2}}\right)+0.15 \theta_{2}^{2} \ln \left(1+\frac{c t}{\theta_{2}^{2}}\right)}
\end{gathered}
$$

Where: $\theta_{1}^{2}=0.533 \mathrm{rad}^{2}, \theta_{2}^{2}=2.85 \mathrm{rad}^{2}, E_{0}$ - electron energy, $t$ - a target thickness in $\mathrm{g} / \mathrm{cm}^{2}$, expressed further through radiating length $X_{0}$ for aluminium, copper and tungsten through factors $24.3,13.0$ and 6.8, accordingly. The constant $c$ is defined for these three elements as $(\pi / 180)^{2} 2.8 \cdot 10^{4},(\pi / 180)^{2} 6.12 \cdot 10^{4},(\pi / 180)^{2} 1.23 \cdot 10^{5}$.
The integrate indicated function in the large importance field is tabulated, and in the small importance field has a kind:

$$
E_{i}=\sum_{m=1}^{100} \frac{(-1)^{m+1} x^{m}}{m ! m}-\ln (x)-0.5772
$$

\section{CHOICE OF MAGNETIC SYSTEM PARAMETERS}

Having limited general length of $6 \mathrm{~m}$, the eight targets variant is considered in the present paper. On which target is lost $10 \%$ of beam energy, so the energy in PMS changes from 200 up to $90 \mathrm{MeV}$. The magnetic field induction in the beginning PMS is chosen close to a limit of electrotechnical steel saturation and equals to $2 \mathrm{~T}$. To keep the beam incidence angular on a target by an energy reduction all magnets are chosen identical on length, and magnetic field induction in the gap are chosen accordingly decreasing. It enables to increase gap height with inevitable growth of beam sizes owing to dispersion on targets. The magnet lengths were choosed proceeding of beam focusing conditions, and in the given variant make $250 \mathrm{~mm}$ with an interval $100 \mathrm{~mm}$. The value of induction and gradients on system length were selected by a consecutive approximate method by the account results proceeding from beam behaviour and beam cross sizes.

\begin{tabular}{|c|c|c|c|c|c|c|c|c|c|c|c|c|c|c|c|}
\hline Cell number & 1 & 2 & 3 & 4 & 5 & 6 & 7 & 8 & 9 & 10 & 11 & 12 & 13 & 14 & 15 \\
\hline Induction, $\mathrm{T}$ & -2 & 2 & -1.8 & 1.8 & -1.58 & 1.58 & -1.4 & 1.4 & -1.26 & 1.26 & -1.14 & 1.14 & -1.04 & 1.04 & 1 \\
\hline Gradient, T/m & 1 & 1 & 0.9 & 0.9 & 0.8 & 0.8 & 0.7 & 0.7 & 0.6 & 0.6 & 0.5 & 0.5 & 0.5 & 0.5 & 0.5 \\
\hline
\end{tabular}

Previously chosen magnetic system parameters are submitted in Table 1.

Table 1: Parameters of periodic magnetic system

\section{TARGET RADIATION CHARACTERISTICS CALCULATED RESULTS}

The calculated results of bremsstrahlung energy integrated angular distribution from all targets PMS, which parameters are given in the table (8 copper targets, initial electron energy $200 \mathrm{MeV}$, energy loss in each of targets make $10 \%$ ), are submitted on Fig.2. The normalised distribution function value $W(\theta)$ for axialsymmetric beam shows, what part of energy is radiated inside of cone with uncover angle $\theta$. As in this case conditions of movement in horizontal and vertical planes are various, the separate functions $W_{x}(\theta)$ and $W_{y}(\theta)$ are used. Ones describes angular distribution in sectors of an elliptic cone located near to axes $o X$ and $o Y$, accordingly. The size $W_{0}(\theta)$ is designed for an ideal hypothetical case when the strictly parallel electron beam falls on each target and is, in essence, unattainable top limit of radiation focusing with the given targets system. Opposite, the function $W_{t}(\theta)$ shows angular distribution from targets in magnetic field absence and, accordingly, beam focusing (total thick target). As it is possible to see from a Fig.2, half of energy from a thick target (stroke- 
dotted curve) is radiated inside of an angle about 2.5 degrees, whereas in an ideal case of parallel beam (dotted curve) this size makes by few more than 0.5 degrees. The functions of distribution in horizontal and vertical planes (continuous curves), designed for the given system, not strongly differ and lay closer to ideal curve.

Half of bremsstrahlung energy in both planes is made in this case in limits about 1 degree. Thus, on distance from system, it is essential greater, than its length, is possible to expect increase of bremsstrahlung flow density in 6-7 times in comparison with a uniform target of equal total thickness.

\section{THE CONCLUSION}

The basic result of the paper given is the physical substantiation of bremsstrahlung density increasing (focusing) method in periodic magnetic system with thin targets, development of account methods of the electron dynamic, radiation characteristics and computer programs. The submitted results are not optimum and can be improved by a more careful choice of the PMS parameters, sizes distributions of an induction and gradients.

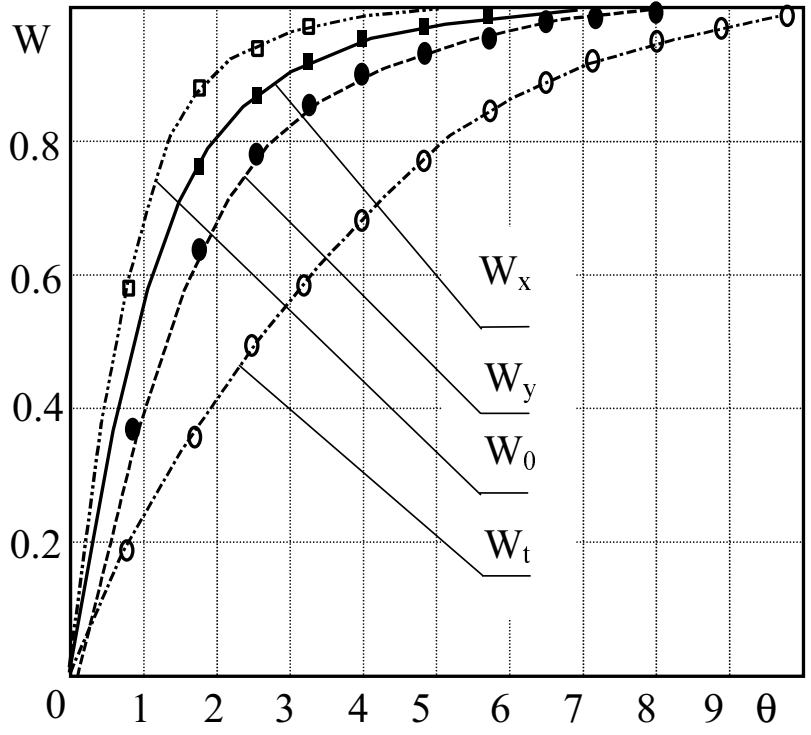

Figure 2 : Integrated angular distribution functions of bremsstrahlung energy from all targets in periodic magnetic system

\section{REFERENCES}

[1] B. Bogdanovitch, A. Nesterovitch, et al. "A Space Accelerator Station for Planet Substance Analysis.” EPAC-98 (6-th Europ. Part. Accel. Conf.), Stockholm, 22-26 June, 1998, Abstracts, p. 37.

[2] B. Bogdanovitch, A. Nesterovitch, et al. " X-ray Radiation Intensity Increasing by means of a Discrete Target in a Magnetic Field. " EPAC-98 (6-th Europ. Part. Accel. Conf.),

Stockholm, 22-26 June, 1998, Abstracts, p. 37.

[3] B. Bogdanovitch, A. Nesterovitch, et al. " Bremsstrahlung Intensity and Average Energy Increase, it's Divergence Reduction in the Target of New Type. " Bull. Of American Phys. Soc., Vol. 42, № 3 (1997), p. 1388. 